\title{
Short-term retention in rats: The effect of goal-arm confinement on delayed alternation performance
}

\author{
DOUGLAS S. GRANT and M. LAYNE MARSHAL \\ University of Alberta, Edmonton, Alberta, Canada
}

\begin{abstract}
Gordon and his associates (e.g., Gordon \& Feldman, 1978, Learning and Motivation, 9, 164-178; Feldman \& Gordon, 1979, Learning and Motivation, 10, 198-210; Gordon, Taylor, \& Mower, 1981, American Journal of Psychology, 94, 309-322) have reported that a reactivation treatment significantly enhances memory for prior forcings in delayed alternation using rats. The reactivation treatment consisted of placing the rat in the goal arm to which it had been forced previously on that trial. The confinement occurred in the absence of food and was $5 \mathrm{sec}$ in duration. The present experiv' :ents explored the possibility that the treatment might influence performance by affording $\because 1$ opportunity for new information to be acquired during the confinement period. Evidence cr, sistent with this view was found in that (1) accuracy was reduced on trials in which the initial event was a 5-sec confinement to the arm opposite that of the target forcing and (2) increasing the duration of the confinement from 5 to 15 to $45 \mathrm{sec}$ increased the magnitude of this effect. It was concluded that the effectiveness of goal-arm confinement as a reactivation treatment does not necessarily implicate processes of retrieval in delayed alternation behavior.
\end{abstract}

Reactivation treatments (often referred to as reminder or pretest cuing treatments) typically involve exposing an organism to a portion of the stimulus complex present during an earlier episode. Such treatments have been found to be effective in reducing normal forgetting over long retention intervals in both pigeons (Moye \& Thomas, 1982) and rats (e.g., Deweer, Sara, \& Hars, 1980; Hamberg \& Spear, 1978). Moreover, reactivation treatments have been shown to reduce forgetting attributable to electroconvulsive shock (Devietti \& Bucy, 1975; Gordon \& Mowrer, 1980), hypothermia (Hinderliter, Webster, \& Riccio, 1975; Mactutus \& Riccio, 1978), inhibition of protein synthesis (Quartermain, McEwen, \& Azmitia, 1970, 1972), immaturity (Spear \& Parsons, 1976), and retroactive interference (Smith \& Spear, 1979). Finally, forgetting caused by prior, conflicting, learning can be enhanced if the memory for that learning is reactivated shortly before training on the target task (Gordon \& Spear, 1973).

The powerful effect of reactivation treatments on longterm retention has led a number of theorists to develop retrieval-based, rather than storage-based, models of memory (e.g., Gordon, 1981; Lewis, 1979; Spear, 1978). The general argument is that if forgetting is a function of information loss, as storage-based models contend, then reactivation treatments should be without effect when ad-

\footnotetext{
This research was supported by Grant A0443 awarded by the Natural Sciences and Engineering Research Council of Canada to the first author. Requests for reprints should be sent to Douglas S. Grant, Department of Psychology, University of Alberta, Edmonton, Alberta, Canada T6G 2E9. M. L. Marshal is presently affiliated with Computing Services, University of Alberta, Edmonton, Alberta.
}

ministered following a source of forgetting, because the original information should no longer be represented in memory. On the other hand, retrieval-based models emphasize reduced accessibilty of information as a major source of forgetting. According to this view, reactivation treatments increase the accessibility of a memory by causing the memory to be reprocessed and associated with contemporary cues.

Gold and King (1974; see also Davis \& Squire, 1984) have suggested, however, that the effectiveness of reactivation treatments in enhancing performance may be irrelevant to the debate between retrieval-based and storagebased models of memory. According to their position, it is possible that such treatments provide an opportunity for new learning which then summates with residual memory of the training episode. To the extent that information acquired during the reactivation treatment is compatible with information acquired during the training episode, enhanced performance at testing would be anticipated by a storage-based model of memory. Notice that this account does not require that the learning presumed to occur during the reactivation treatment be sufficient to support performance in the absence of prior learning. Instead, the account requires only that the level of learning during the reactivation treatment be sufficient to augment a residual, and possibly subthreshold, memory of prior training.

The new learning interpretation of Gold and King cannot be easily dismissed, because most researchers employing reactivation treatments have been satisfied merely to demonstrate that the treatment is (1) effective given prior learning and (2) either without effect or with reduced effect in the absence of prior learning. Such a demonstra- 
tion does not preclude an interpretation in terms of Gold and King's new learning account. Recently, however, Gordon and Mowrer (1980) obtained evidence suggesting that the Gold and King position is at best an incomplete account of reactivation effects on long-term retention. They demonstrated that a reactivation treatment consisting of an extinction trial alleviates amnesia produced by electroconvulsive shock administered shortly after original training. The rationale in using an extinction trial as a reactivation treatment is that any learning that might occur during the treatment should depress performance on a test of memory for the original learning episode. Indeed, animals given original training not followed by electroconvulsive shock demonstrated reduced, rather than enhanced, retention as a function of reactivation. These findings suggest that a reactivation treatment can be effective in alleviating forgetting under conditions that render implausible an account in terms of processes of new learning and summation with residual memory.

The new learning and summation account has not, however, been adequately addressed in recent studies concerning reactivation effects in delayed alternation conducted by Gordon and his associates. The delayed alternation procedure has been employed extensively in the analysis of short-term retention in rats (e.g., Gordon, Brennan, \& Schlesinger, 1976; Grant, 1980, 1981, 1982; Roberts, 1974; see Grant, in press, for a review). Trials involve an initial forcing to one arm of a T-maze. Following a retention interval of a few seconds, a free-choice or test run is administered on which a turn in either direction is permitted. Reinforcement on the test run is contingent upon a turn in the direction opposite that of the forced turn. The reactivation treatment employed by Feldman and Gordon (1979) and Gordon, Taylor, and Mowrer (1981) consisted of interpolating, during the retention interval, a 5-sec confinement in the arm of the maze to which the rat had been forced previously on that trial. The reactivation treatment significantly reduced forgetting in both series of experiments.

In the initial study demonstrating the effectiveness of reactivation in modifying performance in delayed alternation, Gordon and Feldman (1978) found that reactivating memory for a prior, conflicting forcing increased proactive interference. In this preparation, some proportion of the delayed alternation trials are modified such that the rat is forced initially to one arm of the maze (interfering forcing) and then to the opposite arm (target forcing) prior to the retention test. Reinforcement on the test run is contingent upon a turn in the direction opposite to that of the most recent, target forcing. Accuracy on such interference trials is reduced relative to that on standard, control trials on which only the target forcing is administered (Gordon et al., 1976; Grant, 1980). Gordon and Feldman demonstrated that interference is more robust when the interfering and target forcing are separated by $10 \mathrm{sec}$ than when separated by $120 \mathrm{sec}$. More importantly, interference on long interforcing interval trials was enhanced significantly by a reactivation treatment ad- ministered shortly before the target forcing. The reactivation treatment consisted of a 5-sec confinement in the arm to which the rat had been forced during the interfering forcings.

A critical question left unanswered by the research of Gordon and his associates concerns the mechanism(s) through which goal-arm confinement influences freechoice performance. Gordon and his associates have consistently favored a retrieval account in which the reactivation treatment is held to trigger retrieval and reprocessing of information derived from an earlier forcing to that arm. It is reasonable to suggest, however, that the confinement treatment might influence performance in a manner consistent with the view of Gold and King (1974) considered earlier. Specifically, the rat must represent information derived from the forced run in order to perform accurately at the time of testing; that is, to alternate on the free-choice run. It is reasonable to suggest that the confinement treatment may provide an opportunity for information compatible with that derived from the forced run to be acquired, and that free-choice performance therefore reflects the summation of information derived from the confinement treatment with residual, and possibly subthreshold, memory derived from the forcing to that arm. If so, the influence of goal-arm confinement on short-term retention does not necessitate adoption of a retrieval-oriented view of delayed alternation performance.

Indeed, inspection of the data of Gordon and his associates provides considerable justification for entertaining the new learning account. For example, Feldman and Gordon (1979, Experiment 2) included control conditions in which a trial consisted of only one or two confinements followed by a free-choice test (i.e., no forcing was administered). Estimating from their Figure 2, animals chose the arm opposite that to which they were confined on $61 \%$ of the occasions when a single confinement preceded the test by $40 \mathrm{sec}$, and on $65 \%$ of the occasions when two confinements, both to the same arm, preceded the test (the first $90 \mathrm{sec}$ before testing and the second $40 \mathrm{sec}$ before testing). Although Feldman and Gordon did not determine whether these values were significantly above chance (50\%), the percentages do suggest that the confinement treatment mimicks, albeit less strongly, the effects of a forced run to that arm. This finding encourages the view that the effectiveness of goal-arm confinement as a reactivation treatment derives from a summation of information derived from the confinement, with residual information derived from the forced run. As a second example, Gordon and Feldman (1978) found that preceding the target forcing by one or two 5 -sec confinements in the opposite arm reduced accuracy by about $6 \%$. Although this reduction was not statistically significant, it does provide further justification for the view that information compatible with that normally derived from a forced run is acquired during the confinement treatment.

Because of the potential importance of the reactivation phenomenon to our understanding of processes of short- 
term retention, it is important that the effectiveness of the confinement treatment be explored in more than one laboratory. The present experiments investigated further the influence of a reactivation treatment using a procedure highly similar to that employed by Gordon and Feldman (1978). We were concerned in particular with whether the effectiveness of goal-arm confinement as a reactivation treatment might derive from the summation of information acquired during the confinement with that acquired during the earlier forced run.

\section{EXPERIMENT 1}

The present experiment replicated the procedures employed by Gordon and Feldman (1978, Experiment 2). Of primary concern was the effect of the confinement treatment in the presence and absence of a prior forcing. Although the treatment might be expected to have greater influence in the presence of a prior forcing to that arm, it was anticipated that the confinement treatment would influence performance in both cases. The present design permitted this hypothesis to be evaluated by means of two orthogonal contrasts, each involving two trial types. Because the hypotheses under test were a priori and unidirectional and the contrasts were orthogonal, they were evaluated by means of one-tailed $t$ tests, as recommended by Kirk (1968).

\section{Method}

Subjects. Ten naive male Sprague-Dawley rats, approximately 75 days old at the beginning of training, were used. The subjects were reduced to and maintained at $85 \%$ of their ad-lib weights.

Apparatus. A wooden T-maze, painted flat gray throughout and measuring $10 \mathrm{~cm}$ wide with sides $10.5 \mathrm{~cm}$ high, was used. The startbox was $15 \mathrm{~cm}$ long, and the stem portion and each arm were $40 \mathrm{~cm}$ long. Clear Plexiglas guillotine doors were mounted at the entrance to each arm and were used to control the direction of the turning response on forced runs. A glass food cup, painted gray and measuring $1.5 \mathrm{~cm}$ high and $5 \mathrm{~cm}$ in diameter, was located in the middle of the floor at the end of each arm.

Procedure. The first two sessions of the experiment were familiarization sessions. On each of these sessions, each animal received two 5-min exploration periods in the maze. During each exploration period, two 45-mg food pellets were available in each food cup.

Training on the alternation task commenced approximately $24 \mathrm{~h}$ following the final familiarization session. During this training, each animal received eight spaced trials per session. The reinforcer used throughout was two 45-mg food pellets. Each trial involved a reinforced forced run to either the right or left arm. After the reward had been consumed, the rat was returned to the startbox and released immediately ( 0 -sec delay) on the free-choice run. Neither arm was blocked on the free-choice run, and reward was present only in the arm on the side opposite to that entered on the forced run. If the animal entered the rewarded arm, a correct response was recorded and the animal was returned to the holding cage following consumption of the reinforcer. If the animal entered the nonrewarded arm, an incorrect response was recorded and the animal was returned to the startbox and allowed an opportunity to enter the rewarded arm. Correction runs were continued until the rewarded arm was chosen. Alternation training continued for six sessions, at which time all animals were alternating with a high degree of accuracy. The correction procedure was discontinued following alternation training.

Approximately $24 \mathrm{~h}$ following the final session of alternation train- ing, testing began on six types of trials identical to those employed by Gordon and Feldman (1978, Experiment 2). One of the trial types, referred to as "target reactivation (TR)" trials by Gordon and Feldman, is not relevant to the issue of present concern and was included only to reduce any discrepancy between our testing sessions and those of Gordon and Feldman (1978, Experiment 2). A trial type similar to their target reactivation trial type was included also in the second experiment in this series. Data obtained in this trial type will not be reported. The sequence of events involved in the five trial types of interest is illustrated in Table 1. Two of the trial types were control trials, designated by a " $\mathrm{C}$ " in Table 1; the remaining three trial types were proactive interference trials, designated by a "P."

On proactive interference trials, two interfering forcings were administered prior to the target forcing. On each interference trial, both interfering forcings were to the arm of the maze opposite that to which the rat was forced on the subsequent, single target forcing. On proactive-interference short-interforcing-interval (PS) trials, the animal spent a 10 -sec interforcing interval in the holding cage following the second interfering forcing (and hence prior to the target forcing). On proactive-interference long-interforcing-interval (PL) trials, the interforcing interval consisted of a 120 -sec confinement in the holding cage. On proactive-interference long-interforcinginterval reactivation (PLR) trials, the 120-sec interforcing interval was followed by a 5 -sec nonreinforced confinement in the arm to which the rat had been forced on the interfering runs. Confinement was initiated by the experimenter's placing the subject about midway in the goal arm, facing the empty food cup. The reactivation treatment was followed by $10 \mathrm{sec}$ in the holding cage, following which the target forcing was administered.

No interfering forcings were administered on the remaining two types of trials. On control (C) trials, only a single target forcing preceded the test. On control reactivation (CR) trials, the single target forcing was preceded by two events. The animal was first confined, for $5 \mathrm{sec}$ and without food, to the arm of the maze opposite that to which it was subsequently forced on the target run. The confinement was followed by $10 \mathrm{sec}$ in the holding cage and then by the target forcing.

On all trials, animals were placed in the holding cage after the target forcing and then immediately removed and placed in the startbox to begin the free-choice, test run. This operation required approximately $3 \mathrm{sec}$. Each animal was tested on each of the six types of trials once in each session; a left turn on the free-choice run was correct on three of the trials, and a right turn was correct on the other three. The intertrial interval was typically 3 to $4 \mathrm{~min}$. The direction of the rewarded test response was balanced within trial types across sessions, and the order in which the trial types were tested varied across sessions. Testing continued for 12 sessions, yielding 120 observations ( 12 per subject) within each trial type.

The running of subjects was carried out by a paid research assistant who had had considerable experience conducting experiments

Table 1

Illustration of the Trial Types Employed in Experiment 1 Trial Events

\begin{tabular}{|c|c|c|c|c|c|c|}
\hline $\begin{array}{l}\text { Trial } \\
\text { Type }\end{array}$ & $\begin{array}{l}\text { Interfering } \\
\text { Forcings }\end{array}$ & $\begin{array}{l}\text { Interforcing } \\
\text { Interval }\end{array}$ & $\begin{array}{c}\text { Reactivation } \\
\text { Treatment }\end{array}$ & $\begin{array}{c}\text { Target } \\
\text { Forcing }\end{array}$ & Delay & Test \\
\hline PS & $\mathrm{RR}$ & $10 \mathrm{~s}$ & & $\mathrm{~L}$ & $3 \mathrm{~s}$ & $\mathrm{R}$ ? \\
\hline PL & RR & $120 \mathrm{~s}$ & & $\mathrm{~L}$ & $3 \mathrm{~s}$ & R? \\
\hline PLR & RR & $120 \mathrm{~s}$ & $(5 s R)-10 s$ & $\mathrm{~L}$ & $3 s$ & $\mathrm{R}$ ? \\
\hline $\mathrm{CR}$ & & & $(5 s R)-10 s$ & $\mathrm{~L}$ & $3 \mathrm{~s}$ & $\mathrm{R}$ ? \\
\hline C & & & & $\mathrm{L}$ & $3 s$ & $\mathrm{R}$ ? \\
\hline
\end{tabular}

Note $-R=$ right forcing. $L=$ left forcing. $R$ ? = free-choice run on which a right turn was reinforced. $(5$ s $R)=$ placement and 5 -sec confinement in the right goal arm. Numbers followed by " $s$ "designate time periods (in seconds) spent in the holding cage. Substituting $R$ for $L$ and $L$ for $R$ throughout the trial events portion of the table would generate the remaining trials employed. 
employing rats and the alternation procedure. The research assistant was naive with respect to the hypotheses under investigation and was not informed of the anticipated outcome.

\section{Results and Discussion}

Percentage of correct free-choice responses in the five trial types of interest is presented in Table 2. For comparison, accuracy in the corresponding trial types employed by Gordon and Feldman (1978, Experiment 2) is also shown. Although Gordon and Feldman obtained slightly higher accuracy on control-trial types and greater interference on proactive-interference-trial types, the two sets of findings are qualitatively similar except in the critical case of the effectiveness of the reactivation treatment on interference trials employing a long interforcing interval. The reactivation treatment significantly enhanced proactive interference in Gordon and Feldman's experiment, whereas the present data revealed no effect of reactivation on amount of interference (PL vs. PLR, $t<1$ ). In both experiments, the confinement treatment tended to reduce accuracy when that treatment was not preceded by a forcing to that arm ( $\mathrm{C}$ vs. $\mathrm{CR}$ ). In the present experiment, the difference between these two trial types was significant $[\mathrm{t}(9)=2.24, \mathrm{p}<.05]$.

These findings only partially replicate those of Gordon and Feldman. Specifically, both experiments found that in the absence of an interfering forcing, a 5-sec confinement to the arm opposite that of the target forcing tends to reduce accuracy. Although this effect was not significant in their experiment, the trend is consistent with a new learning and summation interpretation of the confinement treatment. Recall that this view does not require that the amount of learning occurring during the reactivation treatment be sufficient to control performance in itself. The two experiments obtained different outcomes, however, with respect to the effect of confinement to the same arm as the interfering forcing. Gordon and Feldman found that such a treatment significantly enhanced proactive interference, whereas the present experiment found that treatment to be without effect. Experiment 2 explored further the effect of the confinement treatment on proactive interference.

\section{EXPERIMENT 2}

Although the preceding experiment was designed to replicate the procedures of Gordon and Feldman (1978, Experiment 2) as precisely as possible, it is clear that they obtained more interference on proactive-interferenc trials involving a short (PS) or long (PL) interforcin. interval than did we in our comparable trial types. In facl accuracy was about $11 \%$ lower in PL than in the contrc (C) trial type in their experiment, whereas accuracy i PL and $\mathrm{C}$ was identical in our Experiment 1. This differ ence suggests that our failure to obtain an effect of th confinement treatment on PLR trials might have resulte from the absence of residual memory for the interferin forcings at the time of the confinement. In Procedures A $B$, and $C$, an attempt was made to increase the strengt of the interfering memory at the time of the confinemer treatment. This was accomplished by reducing the longe interforcing interval from 120 to $60 \mathrm{sec}$ and by increas ing progressively the number of interfering forcings acros the three procedures.

In addition to parametric differences, Experiment 2 dit fered from Experiment 1 in three aspects. First, a differ ent experimenter (the second author) ran the subjects. Sec ond, a different sample of rats was employed. Finally the procedure was streamlined somewhat by eliminatin placements in the holding cage (the interforcing interva was spent in the startbox).

\section{Method}

Subjects. Ten naive male Sprague-Dawley rats, approximatel 75 days old at the beginning of training, were used. The subjec were reduced to and maintained at $85 \%$ of their ad-lib weight: Apparatus. Same as in Experiment 1.

Procedure. Familiarization training was identical to that in Es periment 1. Alternation training was identical to that in Exper ment 1 , except that five, rather than six, sessions were conductec Each of the three procedures described below involved sessions con prising six trials, and each procedure was in effect for 12 sessions yielding 120 observations ( 12 per subject) within each trial typt

Procedure A. Five of the six trial types employed in testing at illustrated in Table 3. [Note: As in Experiment 1, a trial type sim lar to that designated as "target reactivation (TR)" by Gordon an Feldman (1978, Experiment 2) was employed in Procedures A, E and $C$ but will not be described further.] The trial types can $t$ described most readily through comparison with those employe in Experiment 1. Comparison of Tables 1 and 3 reveals that all placi ments in the holding cage were eliminated. Therefore, immediatel after consuming the reinforcer on the target, forcing animals we1 placed in the startbox and released on the free-choice run. This ope ation required about $1 \mathrm{sec}$ rather than $3 \mathrm{sec}$. Also, there were n holding-cage placements following either reactivation treatmen or initial forcings. Two other changes were that only one interfe1 ing forcing was administered and that the longer interforcing ir terval was $60 \mathrm{sec}$ rather than $120 \mathrm{sec}$. Except for these modific: tions, the trial types were identical to those in Experiment 1

Table 2

Percentage of Correct Responses as a Function of Trial Type in Experiment 1

\begin{tabular}{lcc}
\hline \multicolumn{1}{c}{ Trial Type } & Experiment 1 & Gordon \& Feldman \\
\hline Proactive Interference: & & \\
Short Interforcing Interval (PS) & 73.4 & 57 (P-10) \\
Long Interforcing Interval (PL) & 89.2 & 83 (P-120) \\
Long Interforcing Interval, Reactivation (PLR) & 90.0 & 62 (ER) \\
Control (C) & 89.2 & 95 (R) \\
Control, Reactivation (CR) & 85.0 & 89 (RC) \\
\hline
\end{tabular}

Note-Accuracy in comparable trial types employed by Gordon and Feldman (1978, Experiment 2) was estimated from their Figure 2. The group designations employed by Gordon and Feldman are enclosed in parentheses. 
Table 3

Illustration of the Trial Types Employed in Experiment $2 A$

\begin{tabular}{|c|c|c|c|c|c|c|}
\hline \multirow[b]{2}{*}{$\begin{array}{l}\text { Trial } \\
\text { Type }\end{array}$} & \multicolumn{6}{|c|}{ Trial Events } \\
\hline & $\begin{array}{l}\text { Interfering } \\
\text { Forcing }\end{array}$ & $\begin{array}{l}\text { Interforcing } \\
\text { Interval }\end{array}$ & $\begin{array}{c}\text { Reactivation } \\
\text { Treatment }\end{array}$ & $\begin{array}{c}\text { Target } \\
\text { Forcing }\end{array}$ & Delay & Test \\
\hline PS & $\mathrm{R}$ & $10 \mathrm{~s}$ & & $\mathrm{~L}$ & $1 \mathrm{~s}$ & $\overline{\mathrm{R}}$ ? \\
\hline PL & $\mathbf{R}$ & $60 \mathrm{~s}$ & & $\mathrm{~L}$ & $1 \mathrm{~s}$ & $\mathrm{R}$ ? \\
\hline PLR & $\mathrm{R}$ & $60 \mathrm{~s}$ & $(5 s R)-0 s$ & $\mathrm{~L}$ & $1 \mathrm{~s}$ & $\mathrm{R}$ ? \\
\hline $\mathrm{CR}$ & & & $(5 s R)-0 s$ & $\mathrm{~L}$ & $1 \mathrm{~s}$ & $\mathrm{R}$ ? \\
\hline $\mathrm{C}$ & & & & $\mathrm{L}$ & $1 \mathrm{~s}$ & $\mathrm{R}$ ? \\
\hline
\end{tabular}

Note $-R=$ right forcing. $L=$ left forcing. $R$ ? = free choice run on which a right turn was reinforced. $(5 s R)=$ placement and 5 -sec confinement in the right goal arm. Numbers followed by " $s$ " designate time periods (in seconds) spent in the startbox. Substituting $R$ for $L$ and $L$ for $R$ throughout the trial events portion of the table would generate the remaining trials employed.

Table 4

Percentage of Correct Responses as a Function of Trial Type in Each of the Procedures of Experiment 2

\begin{tabular}{lccc}
\hline \multicolumn{1}{c}{ Trial Type } & $2 \mathrm{~A}$ & $2 \mathrm{~B}$ & $2 \mathrm{C}$ \\
\hline Proactive Interference: & & & \\
Short Interforcing Interval (PS) & 73.3 & 75.0 & 57.5 \\
Long Interforcing Interval (PL) & 90.8 & 94.1 & 86.7 \\
Long Interforcing Interval, Reactivation (PLR) & 89.1 & 90.8 & 76.7 \\
Control (C) & 89.2 & 91.6 & 88.3 \\
Control, Reactivation (CR) & 85.0 & 88.3 & 82.5 \\
\hline
\end{tabular}

Procedure B. Approximately $24 \mathrm{~h}$ following completion of Procedure $A$, the subjects began testing on Procedure $B$. The two controltrial types were identical to those in the preceding procedure. The three proactive-inteference-trial types differed only in that two interfering forcings were administered in immediate succession. All other aspects of the procedure were the same as in Procedure A.

Procedure C. Approximately $24 \mathrm{~h}$ following completion of Procedure $B$, each animal was given six sessions of alternation training. This training was identical to that described earlier, except that 10 , rather than 8 , trials composed a session. Approximately $24 \mathrm{~h}$ following the sixth alternation session, testing on Procedure $\mathrm{C}$ began. Control-trial types were identical to those employed in Procedures $\mathrm{A}$ and $\mathrm{B}$. The proactive-interference-trial types differed only in that three interfering forcings were administered. All other aspects of the procedure were the same as in Procedure A.

\section{Results and Discussion}

Percentage of correct responses in each of the five trial types of interest in each of the three procedures is shown in Table 4 . Accuracy was reduced by confining the animal in the arm opposite to that of the subsequent target forcing in all three procedures, although accuracy on $\mathrm{C}$ trials was significantly higher than that on CR trials only in Procedure A $[t(9)=3.04, p<.01]$. Accuracy was also reduced by confining the animal to the same arm as the interfering forcing, although accuracy on PL trials was significantly higher than that on PLR trials only in Procedure $C[t(9)=2.09, p<.05]$. Notice that relative to Procedures A and B, accuracy on proactive interference trials involving either a short (PS) or long (PL) interforcing interval was reduced more markedly in Procedure $\mathrm{C}$. The latter finding suggests that a 5-sec confinement may enhance proactive interference only under conditions in which residual memory for the interfering forcing(s) is present at the time of the confinement treatment.

\section{EXPERIMENT 3}

Although the preceding experiments revealed that a confinement to the arm opposite to that of the target forcing tends to reduce accuracy in the absence of a recent forcing to that arm ( $\mathrm{C}$ vs. CR trials), the magnitude of the effect was small and attained significance in only two of four instances. Recall, however, that a new learning and summation view does not require that the reactivation treatment afford an opportunity for the acquisition of information sufficient to control performance. Rather, what is required is that enough information be acquired during the treatment to result in a suprathreshold memory when information derived from the reactivation treatment is combined with residual information derived from the forcing. It does seem reasonable to argue that if a 5-sec confinement is sufficient to result in the acquisition of some new information, then longer confinements should result in the acquisition of even more new information. If so, increasing the duration of the confinement treatment should result in the treatment's approximating more closely the effect of an actual forced run.

To test this notion, only the two types of control trials were employed and rats were confined on different $C R$ trials for 5,15 , or $45 \mathrm{sec}$. It was anticipated that longer confinement would afford an opportunity for more information to be acquired than would a shorter confinement. If so, a longer confinement treatment should more closely approximate the effect of a forced run. Because the confinement treatment was administered in the goal arm opposite that to which the animal was forced on the target forcing on CR trials, accuracy should be attenuated more markedly on CR trials involving confinement of longer duration.

\section{Method}

Subjects. Eight naive male Sprague-Dawley rats, approximately 75 days old at the beginning of training, were used. The subjects were reduced to and maintained at $85 \%$ of their ad-lib weights.

Apparatus. Same as in Experiment 1.

Procedure. Familiarization and alternation training were identical to that in Experiment 1. Following alternation training, the animals were tested on $\mathrm{C}$ and $\mathrm{CR}$ trials. Thus, no interfering forcings were administered in this experiment. The two control trial types were identical to those of Experiment 2 (see Table 3), except that the duration of confinement on different CR trials was 5,15 , or $45 \mathrm{sec}$. Each testing session consisted of eight trials, two of each type (one involving a correct free-choice turn to the right, and the other a correct free-choice turn to the left). The order of testing trials within a session varied from session to session. Testing continued for 20 sessions, yielding 320 observations (40 per subject) within each of the four trial types.

The running of subjects was carried out by a paid research assistant who had had considerable experience in conducting experiments employing rats and the delayed alternation procedure. The research assistant was naive with respect to the hypothesis under investigation and was not informed of the anticipated outcome. Although a paid research assistant was employed to conduct both Experiments $I$ and 3, a different assistant was used for each experiment 


\section{Results and Discussion}

Percentage of correct responses on C, CR-5, CR-15, and CR-45 trials was $95.3,90.6,87.1$, and 80.1, respectively. A one-way analysis of variance on data from all four conditions revealed a significant effect of conditions $[F(3,21)=27.90, p<.001]$, as did a similar analysis performed on data from the three CR conditions $[\mathrm{F}(2,14)$ $=229.37, \mathrm{p}<.001]$. Newman-Keuls tests $(\alpha=.05)$ revealed the ordering of conditions to be control $>C R-5$ $=$ CR-15 > CR-45. Thus, alternation accuracy was higher on $C$ trials than on any of the three CR-trial types. In addition, accuracy was depressed more markedly by the confinement treatment when that treatment was $45 \mathrm{sec}$ in duration than when it was either 5 or $15 \mathrm{sec}$ in duration. These data are consistent with the hypotheses that (1) information similar to that acquired during a forced run is acquired during a goal-arm confinement and (2) the longer the confinement, the greater is the amount of information acquired. These results are clearly consistent with a new learning interpretation of the effectiveness of goal-arm confinement as a reactivation treatment in the delayed alternation preparation.

\section{GENERAL DISCUSSION}

The present experiments provide considerable support for a new learning and summation interpretation of the effectiveness of goal-arm confinement as a reactivation treatment in the delayed alternation preparation. The notion that goal-arm confinement provides an opportunity for the acquisition of information that is similar to that acquired during a forced run is strongly supported by the finding that a confinement treatment administered in the absence of a recent forcing to that arm on control reactivation trials has an effect similar to, albeit less powerful than, the effect of a forcing to that arm. Indeed, accuracy on control reactivation trials involving a 5 -sec confinement was lower than that on control trials in all five instances and statistically lower in three of the five cases. Failure to detect a statistically reliable effect of the 5-sec confinement on control reactivation trials in some cases in Experiments 1 and 2 might reflect, at least in part, an insufficient number of observations. Because our first two experiments were patterned after Gordon and Feldman's (1978) second experiment, we followed their procedure of testing each subject on only one trial of each type per session. We did, however, employ two additional subjects (10 vs. 8 ) and two additional testing sessions (12 vs. 10). Thus, data reported in Experiments 1, 2A, 2B, and $2 \mathrm{C}$ were based on 120 observations (12 per subject), whereas those reported by Gordon and Feldman in their second experiment were based on only 80 observations (10 per subject). Nevertheless, 120 observations may still be insufficient to permit statistical detection of a reduction in accuracy of five or six percentage points on control reactivation trials.

Recall again, however, that the new learning and summation view does not require that the confinement treat- ment result in the acquisition of information sufficient to control performance in the absence of residual information from a prior forcing. Thus, whether or not the confinement treatment administered on control reactivation trials lowers accuracy is not critical. On the other hand, it is critically important that the confinement treatment provide an opportunity for the acquisition of new information compatible with that derived from a forcing. Although the finding that the 5-sec confinement administered on control reactivation trials tends to lower accuracy is consistent with the new learning and summation view, more important was the finding in Experiment 3 that increasing the duration of the confinement from 5 to 15 to $45 \mathrm{sec}$ enhanced the difference in accuracy on control and control reactivation trials. This finding suggests strongly that a confinement in a goal arm produces effects similar to, although less powerful than, an actual forced run to that arm. To complete the new learning account of the effectiveness of goal-arm confinement as a reactivation treatment, it is necessary only to add the theoretically noncontentious assumption that the information derived from the confinement treatment summates with residual memory for information derived from the forced run(s). It is important to note that the present findings do not preclude the possibility that the confinement treatment might result in the retrieval and reprocessing of memory for a prior forcing. However, the new learning and summation interpretation provides a more complete and parsimonious account of the available data.

Regardless of the ultimate theoretical account of the effect of goal-arm confinement on delayed alternation performance, two conclusions are possible. First, the data of Gordon and his associates and especially the present data suggest rather strongly that the confinement treatment does influence accuracy in the absence of a recent forcing to that arm. We have interpreted this result as reflecting the acquisition of new information during the confinement treatement, information that is similar to that derived from a forced run. Second, a corollary of the first conclusion is that the reactivation phenomenon may tell us little of theoretical interest about short-term retention in rats. That is, unless it can be shown that a treatment produces opposite effects on performance in the presence and absence of a prior memory, the effectiveness of such a treatment is theoretically indecisive. Such a demonstration has yet to occur in the delayed alternation preparation, and, therefore, whether the treatment influences retention through processes of new learning and summation and/or memory retrieval and reprocessing remains unclear.

\section{REFERENCES}

Davis, H. P., \& SQuire, L. R. (1984). Protein synthesis and memory: A review. Psychological Bulletin, 96, 518-559.

DeVIEtTI, T. L., \& BUCY, C. E. (1975). Recovery of memory after reminder: Evidence for two forms of retrieval deficit induced by ECS. Physiological Psychology, 3, 19-25.

Deweer, B., Sara, S. J., \& Hars, B. (1980). Contextual cues and memory retrieval in rats: Alleviation of forgetting by a pretest ex- 
posure to background stimuli. Animal Learning \& Behavior, 8, 265-272.

Feldman, D. T., \& Gordon, W. C. (1979). The alleviation of shortterm retention decrements with reactivation. Learning and Motivation, 10, 198-210.

GoLD, P. E., \& KING, R. A. (1974). Retrograde amnesia: Storage failure versus retrieval failure. Psychological Review, 81, 465-469.

GoRDON, W. C. (1981). Mechanisms of cue-induced retention enhancement. In N. E. Spear \& R. R. Miller (Eds.), Information processing in animals: Memory mechanisms (pp. 319-339). Hillsdale, NJ: Erlbaum.

Gordon, W. C., Brennan, M. J., \& Schlesinger, J. L. (1976). The interaction of memories in the rat: Effects on short-term retention performance. Learning and Motivation, 7, 406-417.

Gordon, W. C., \& Feldman, D. T. (1978). Reactivation-induced interference in a short-term retention paradigm. Learning and Motivation, 9, 164-178.

Gordon, W. C., \& Mowrer, R. R. (1980). An extinction trial as a reminder treatment following electroconvulsive shock. Animal Learming \& Behavior, 8, 363-367.

Gordon, W. C., \& SPEAR, N. E. (1973). The effect of reactivation of a previously acquired memory on the interaction between memories in the rat. Journal of Experimental Psychology, 99, 349-355.

GoRDON, W. C., TAYLOR, J. R., \& MOWRER, R. R. (1981). Enhancement of short-term retention in rats with pretest cues: Effects of the training-cueing interval and the specific cueing treatment. American Journal of Psychology, 94, 309-322.

Grant, D. S. (1980). Delayed alternation in the rat: Effect of contextual stimuli on proactive interference. Learning and Motivation, 11, 339-354.

GRANT, D. S. (1981). Intertrial interference in rat short-term memory. Journal of Experimental Psychology: Animal Behavior Processes, 7, 217-227.

GRANT, D. S. (1982). Stimulus control of information processing in rat short-term memory. Journal of Experimental Psychology: Animal Behavior Processes, 8, 154-164.

GRANT, D. S. (in press). Delayed alternation and short-term retention in the rat. In D. F. Kendrick, M. Rilling, \& M. R. Denny (Eds.), Theories of animal memory. Hillsdale, NJ: Erlbaum.
Hamberg, J. M., \& SPEAR, N. E. (1978). Alleviation of forgetting of discrimination learning. Learning and Motivation, 9, 466-476.

Hinderliter, C. F., Webster, T., \& Riccio, D. C. (1975). Amnesia induced by hypothermia as a function of treatment-test interval and recooling in rats. Animal Learning \& Behavior, 3, 257-263.

KIRK, R. E. (1968). Experimental design: Procedures for the behavioral sciences. Belmont, CA: Wadsworth.

LewIS, D. J. (1979). Psychobiology of active and inactive memory. Psychological Bulletin, 86, 1054-1083.

MACTUTUS, C. F., \& RICCIO, D. C. (1978). Hypothermia-induced retrograde amnesia: Role of body temperature in memory retrieval. Physiological Psychology, 6, 18-22.

Moye, T. B., \& ThOMAs, D. R. (1982). Effects of memory reactivation treatments on postdiscrimination generalization performance in pigeons. Animal Learning \& Behavior, 10, 159-166.

Quartermain, D., McEwen, B. S., \& Azmitia, E. C. (1970). Amnesia produced by electroconvulsive shock or cycloheximide: Conditions for recovery. Science, 169, 683-686.

Quartermain, D., McEwen, B. S., \& Azmitia, E. C. (1972). Recovery of memory following amnesia in the rat and mouse. Journal of Comparative and Physiological Psychology, 79, 360-370.

ROBERTS, W. A. (1974). Spaced repetition facilitates short-term retention in the rat. Journal of Comparative and Physiological Psychology, 86, 164-171.

SMITH, G. J., \& SPEAr, N. E. (1979). Reactivation of an appetitive discrimination memory following retroactive interference. Animal Learming and Behavior, 7, 289-293.

SPEAR, N. E. (1978). The processing of memories: Forgetting and retention. Hillsdale, NJ: Erlbaum.

SPEAR, N. E., \& PARsons, P. J. (1976). Analysis of a reactivation treatment: Ontogenetic determinants of alleviated forgetting. In D. L. Medin, W. A. Roberts, \& R. T. Davis (Eds.), Processes of animal memory (pp. 135-165). Hillsdale, NJ: Erlbaum.

(Manuscript received July 26, 1984; revision accepted for publication January 23, 1985.) 